\title{
La Sociedad de la Información en los países árabes: una aproxi- mación al análisis de indicadores socioeconómicos
}

\author{
Khaldoon Al Dwairi \\ Victor Herrera-Solana *
}

Artículo recibido:

16 de febrero de 2007.

Artículo aceptado:

3 de mayo de 2007.

\section{RESUMEN}

En el presente artículo mostramos el nivel de lo que se suele llamar Sociedad de la Información en los países árabes. Se presentan los problemas que han encontrado las nuevas tecnologías para su implantación y evolución en esta zona; cuál ha sido la postura de los diferentes países frente a las posibilidades ofrecidas por Internet, desde su origen hasta la situación actual; los problemas que Internet ha tenido para desarrollarse en la región, y las diferencias que entre cada uno de estos países se pueden apreciar.

Se muestra el nivel de infraestructura de las nuevas tecnologías, revelando tanto los indicadores socioeconómicos (población, densidad, renta per cápita, nivel

* Los dos autores pertenecen a la Universidad de Granada, España, (Khaldoon: khaldoc@ugr.es); (Enrique: victorhs@ugr.es).

INVESTIGACIÓN BiBLIOTECOLÓGICA, Vol. 21, Núm. 43, julio/diciembre, 2007, México, ISSN: 0187-358X. pp. 185-208 
de alfabetización) como los indicadores tecnológicos (líneas telefónicas, ordenadores, proveedores, servidores, usuarios).

El estudio muestra el nivel de desarrollo de cada uno de los países, los cuales clasificamos en tres grandes grupos en función de su riqueza. Finalmente se muestra cómo estos grupos no son cerrados y cómo hay países que pueden incluirse en más de un grupo.

Palabras Clave: Sociedad de la Información; Países árabes; Internet.

\section{ABSTRACT}

The Information Society in the Arab Countries: an approach to socio-economic indicators' analysis

Khaldoon Al Dwairi and Victor Herrera-Solana

The situation of Information Science in Arab Countries is described, and problems related to the implementation of new information technologies in the region, also considered, as well as the attitude of each one of these countries towards Internet. Socio-economic (population, density, per capita income, literacy) and technological indicators (number of telephone lines, computers, ISPs, servers, users) are presented together with the level of development of each country. These countries are divided into three different economic groups (A,B and C), but results show that such groups are not clearly delineated with respect to technological incorporation in society.

Keywords: Information society; Arab World; Internet.

\section{INTRODUCCIÓN}

\section{1. ¿Qué son las Tecnologías de la Información y la Comunicación (TIC)?}

T as TIC son el conjunto de tecnologías que permiten transmitir, procesar Ly difundir la información de manera rápida y automática, siendo éstas la base sobre la cual se crea la "Sociedad de la Información". Se describe ésta como una sociedad en la que ... 
todos puedan crear, acceder, utilizar y compartir la información y el conocimiento, para hacer que las personas, las comunidades y los pueblos puedan desarrollar su pleno potencial y mejorar la calidad de sus vidas de manera sostenible". ${ }^{1}$

Para medir y evaluar el acceso de las personas a las (TIC) se estudian algunos indicadores sobre el nivel de infraestructura que tiene un país. Con este nivel pueden medirse variables tales como el número de líneas telefónicas principales, e indicadores de acceso y utilización a éstas. Por ejemplo, casi todos los países del mundo tienen conexión directa a Internet como refleja la Figura 1. Entre 2000 y 2002, no hubo ningún país que se conectara a Internet. En septiembre de 2003 la Isla de Tokelau fue el último país en conectarse a Internet.

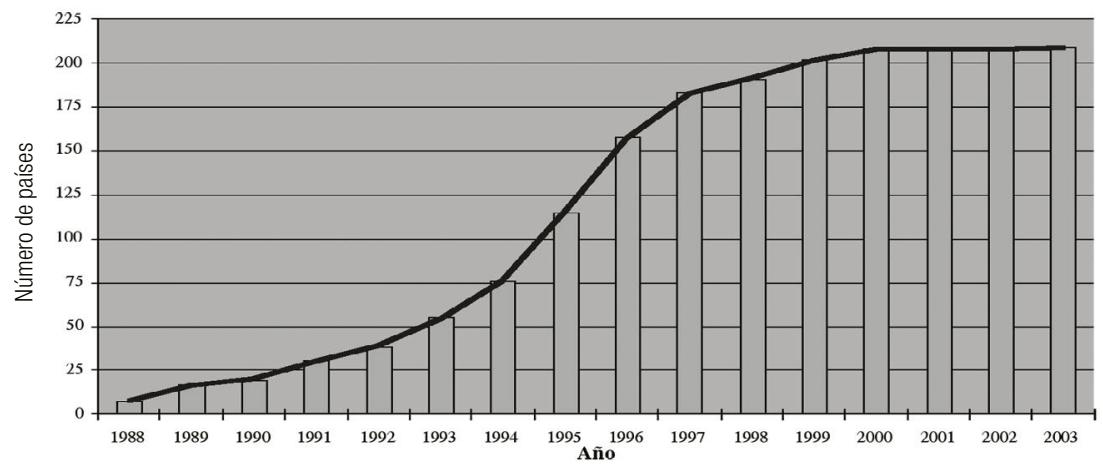

Fig.1: Número de países con conexión directa a Internet 1988-2003.

Fuente: Elaboración propia a partir de los datos de la Unión Internacional de Telecomunicaciones (ITU).

Los niveles de penetración de las TIC varían de un país a otro en función del nivel de desarrollo tecnológico que cada uno tenga, cómo también de su nivel cultural y adquisitivo. Esto genera grandes diferencias y ha dado lugar a lo que suele conocerse como brecha digital. En los países desarrollados se suelen realizar muchas mediciones que involucran un gran número de factores tales como: la infraestructura, la utilización, el volumen y el valor de las TIC, así como el acceso a ellas; por el contrario, los países en desarrollo tienen muy pocos indicadores básicos.

Pero ¿quién tiene acceso a las TIC? Parte de la respuesta dependerá de los indicadores implicados. Lo más tradicional consiste en dividir el número de

1 Cumbre Mundial sobre la Sociedad de la Información, en línea], Unión Internacional de Telecomunicaciones. Actualizado el 17 de diciembre del 2004. [Última consulta el 25 del 02 del 2005] Disponible en:

<http://www.itu.int/wsis/index-es.html>. 
dispositivos o servicios de acceso entre el número total de habitantes. Si bien este resultado per cápita es conveniente y útil para comparar las diferencias generales entre los países (incluso dentro de ellos), puede inducir a error, debido a que el indicador per cápita no refleja la composición sociodemográfica.. En los resultados per cápita tampoco se tienen en cuenta los principios de distribución, por ejemplo, el número de teléfonos en los hogares, o de ordenadores en cibercafés y empresas. En los resultados por hogares tampoco se tiene presente el acceso a las TIC desde el lugar de trabajo, la escuela o a través de iniciativas gubernamentales.

De acuerdo con el Informe sobre el desarrollo mundial de las telecomunicaciones del 2003², debe establecerse una diferencia entre TIC antiguas (receptores de radio, televisión y teléfonos fijos) y TIC nuevas (teléfonos móviles, ordenadores personales y conexiones a Internet). La mayoría de los países en desarrollo tienden a disponer de datos sobre las TIC antiguas, mientras que casi todos los países desarrollados suelen centrarse en las más nuevas.

Las TIC más recientes tienen, además, mayor tendencia a depender de la disponibilidad de energía o de instalaciones de recarga, lo que parece indicar que el porcentaje de hogares con electricidad es un indicador decisivo para medir las posibilidades de acceso a las TIC en los países en desarrollo. Las TIC, por tanto, son un bien consumible no disponible en todos los países en desarrollo.

\subsection{Estado de Internet en el mundo árabe}

Mientras que Internet se ha desarrollado en el mundo occidental durante los últimos años, su presencia como parte de la vida cotidiana en el mundo árabe es relativamente nueva. Las primeras conexiones a Internet en estos países datan de principios de los años 90. Túnez fue el primer país árabe en tener conexión a Internet (1991), y Kuwait estableció servicios de Internet en 1992 como parte de su reconstrucción después de la invasión iraquí. En 1993, Egipto y los EAU (Emiratos Árabes Unidos) establecieron conexión a Internet, mientras que Jordania se unió a Internet en 1994. y Somalia y Arabia Saudí lo hicieron a finales de la década de los 90. En la Figura 2 se puede observar el año en el que se conectaron a Internet los diferentes países de la región.

2 Informe sobre el Desarrollo Mundial de las Telecomunicaciones 2003: Indicadores de acceso para la sociedad de la información, en línea]. International Telecommunication Union. 2003. [Última consulta el: 26 del 09 del 2004]. Disponible en: 


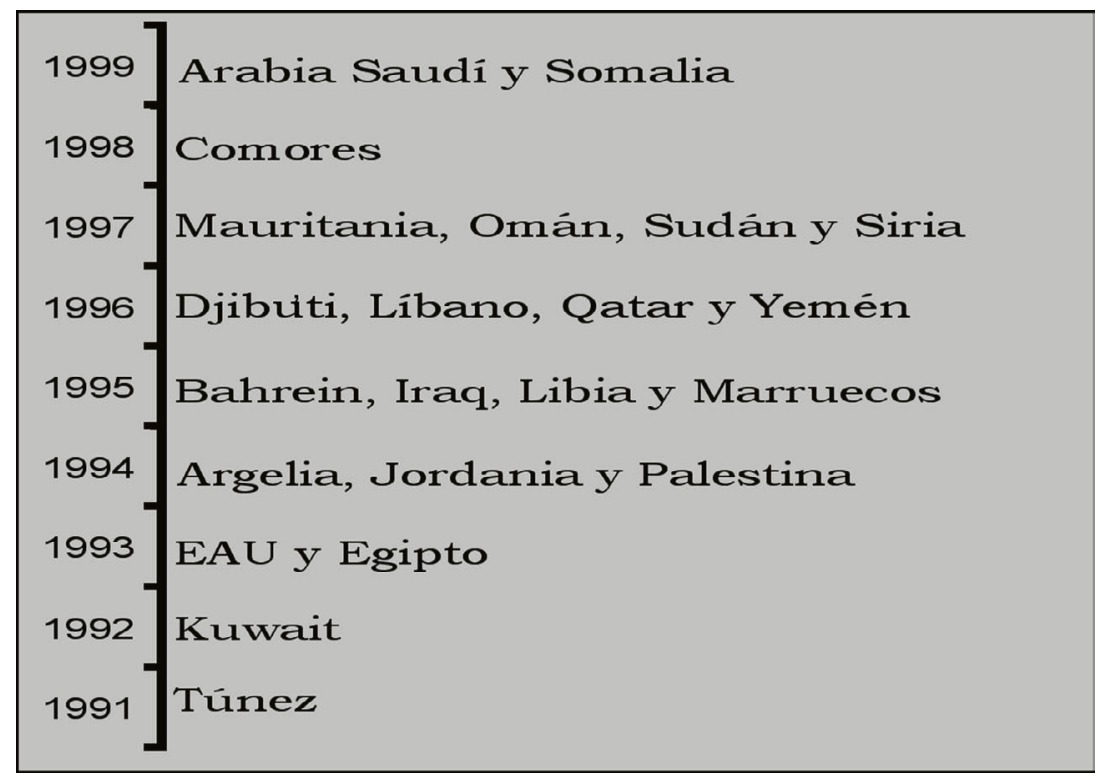

Fig. 2: Año de acceso a Internet de los países de la Región MENA. ${ }^{3}$

Fuente: Elaboración propia

En general, en los países con nivel económico alto este acceso es más fácil pero en este gráfico se observa que Túnez, un país que no tiene un nivel económico alto, como es el caso de Arabia Saudí, fue no obstante el primer país que obtuvo el accceso. Probablemente a Túnez le interesaba tener acceso por las ventajas económicas que ello le podía aportar. Por el contrario, Arabia Saudí ha sido uno de los últimos países de la región en tener acceso a Internet, posiblemente por sentir que sus valores morales se verían amenazados con tal acceso.

En la región MENA la difusión de Internet parece aumentar más lentamente que en el resto del mundo, debido a que los cambios económicos, políticos y sociales han encontrado muchos inconvenientes. La visión general sobre el acceso y el uso público de Internet no refleja el hecho de que la mayoría de los usuarios de la región obtiene el acceso a través de cibercafés, empresas o universidades, y no con una conexión en su hogar. En la siguiente (Tabla 1) se aprecian datos globales sobre el número de usuarios de Internet distribuidos por grandes regiones mundiales.

3 MENA: Middle East and North of Africa, en este trabajo se usará indistintamente cómo sinónimo del Mundo Árabe. 
Tabla I: Usuarios de Internet por regiones en millones de habitantes en el 2004.

\begin{tabular}{|c|c|}
\hline Total del mundo & $\mathbf{6 0 5 , 6 0}$ Millones \\
\hline África & 6,31 Millones \\
\hline Asia / Pacífico & 187,24 Millones \\
\hline Europa & 190,91 Millones \\
\hline Oriente Medio & 5,12 Millones \\
\hline Canadá y EE.UU. & 182,67 Millones \\
\hline América Latina & 33,35 Millones \\
\hline
\end{tabular}

Fuente: UNA ${ }^{4}$.

Según el Royal Institute for Inter-Faith Studies, si se suman todos los usuarios de Internet de la sociedad árabe, se obtiene un total de 4.902.200 usuarios de la Internet en la zona MENA. Éstos constituyen solamente el 0,75\% de los 600 millones usuarios de Internet en todo el mundo. Por lo tanto, la región, que constituye el $4 \%$ de la población del mundo, tiene menos del 1\% de los usuarios de Internet. ${ }^{5}$ Se ve claramente que el uso de Internet en el mundo árabe es generalmente inferior a la media mundial. Sin embargo no hay que generalizar porque existen grandes diferencias entre los propios países árabes, y también causas externas que ayudan a entender esta situación, en particular los embargos impuestos a determinados países: Libia, Iraq y Sudán, así como, el aislamiento político a Siria.

Sin embargo los factores internos son más importantes, en particular las prohibiciones que los gobiernos de los países árabes les imponen a sus habitantes. En primer lugar, Internet llegó tarde a algunos países, en parte debido a recelos contra todo lo que provenga de Occidente, y en parte, porque hay gobiernos que perciben a Internet como una amenaza contra las costumbres y tradiciones locales. Asimismo, determinados gobiernos están acostumbrados a controlar la información de la que disponen sus ciudadanos, y se sienten amenazados por el libre acceso a todo tipo de información que supone la Internet. En cuanto al ciudadano, éste encuentra otros obstáculos además de los políticos, económicos o administrativos, como es el hecho de que el porcentaje de información disponible en Internet en lengua árabe todavía es muy inferior al disponible en otros idiomas.

4 Jupitermedia Corporation. NUA internet how many online, [en línea], 2004. [Última consulta el 11 del 05 del 2004]. Disponible en:

$<$ http://www.nua.com/surveys/how_many_online/>.

5 The Arabic Network for Human Rights Information. The Internet In the Arab World A New Space of Repression?: Overview, [en línea], 2003-2004; [Última consulta el 16 del 11 del 2004]. Disponible en: 


\subsection{Objetivo}

El objetivo de este trabajo es recopilar información relativa a diferentes parámetros para poder explicar la evolución de Internet en los Países Árabes. Mostraremos las diferencias en la implantación y acceso a Internet que existen entre los distintos países a través de diferentes indicadores, tanto socioeconómicos como tecnológicos. Los factores concretos que se van a analizar en este trabajo son los siguientes:

Indicadores socioeconómicos:

1. La densidad de población.

2. La renta per cápita y la riqueza del país.

3. El nivel de alfabetización de los países.

Indicadores tecnológicos:

4. Número de líneas telefónicas por cada 100 habitantes.

5. Número de ordenadores por cada 100 habitantes.

6. Proveedores de servicios de Internet existentes (ISP).

7. Número de servidores por cada 10.000 habitantes.

8. Número de usuarios de Internet por cada 10.000 habitantes.

\section{Material y MÉtodo}

Se considera Mundo Árabe Middle East and North of Africa (MENA) a aquellos países cuyo idioma oficial es el árabe; a este mundo lo forman veintidós países: Arabia Saudí, Argelia, Bahrein, las Islas de Comores (Comores), Dijbuti, Egipto, Emiratos Árabes Unidos (EAU), Iraq, Jordania, Kuwait, Líbano, Libia, Marruecos, Mauritania, Omán, Palestina, Qatar, Siria, Somalia, Sudán, Túnez y Yemen. Irán y Turquía no se consideren países árabes porque sus idiomas oficiales son el Farsi y el Turco respectivamente. Los Países Árabes tienen una rica diversidad de comunidades étnicas, lingüísticas y religiosas. 


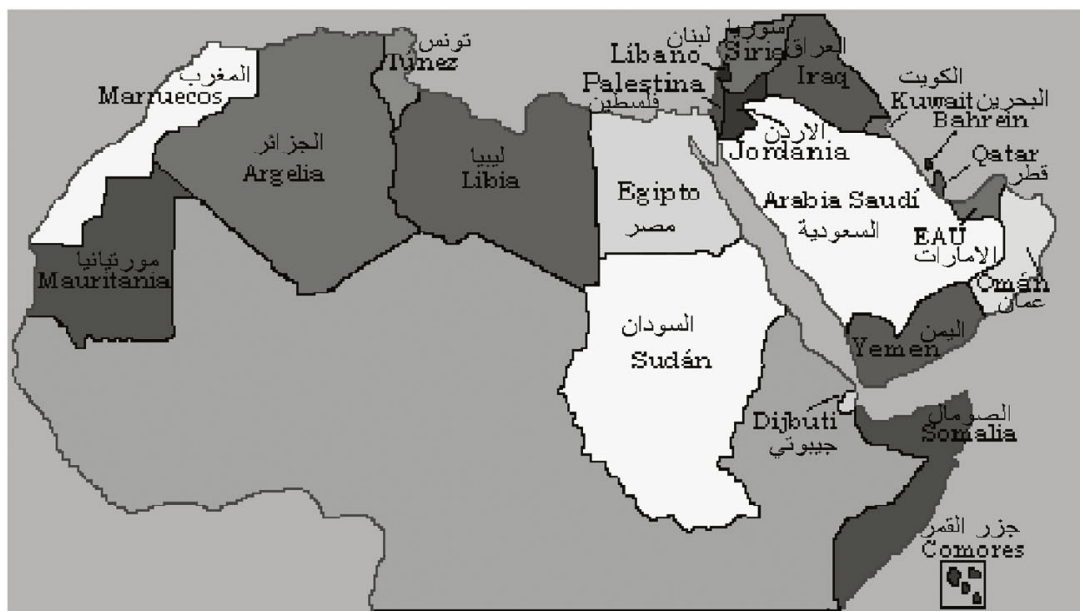

Fig. 3. Países que constituyen la Región MENA ${ }^{6}$

Fuente: Elaboración propia

Los Países Árabes se encuentran formando parte de diferentes regiones geográficas, y según éstas podemos clasificarlos del siguiente modo:

- Oriente Medio (Estados Árabes del Este), formado por siete países: Egipto, Iraq, Jordania, Líbano, Siria, Palestina y Yemen

- Estados Árabes Africanos, que comprende nueve países: Argelia, Comores, Dijbuti, Libia, Marruecos, Mauritania, Somalia, Sudán y Túnez

- Estados del Golfo Pérsico, que incluye seis países: Arabia Saudí, Bahrein, Emiratos Árabes Unidos (EAU), Kuwait, Omán y Qatar

Egipto se localiza físicamente en el norte de África pero para todos los efectos forma parte de Oriente Medio. Yemén, que se encuentra en la zona del Golfo, no pertenece a ellos, debido principalmente a la ausencia de petróleo en su territorio, mientras que el resto de los países de la península sí lo tienen. Éstos formaron el Consejo de Cooperación de los Países del Golfo Árabe, y mantienen entre ellos un sistema de mercado libre. Por otra parte, hay que tener en cuenta la situación especial de algunos países como Somalia, que vive en estado de caos social y económico, y de otros dos países que viven en estado de guerra de facto: Iraq y Palestina. En estos casos en particular

6 Intersol The Global Advisor Newsletter - Arabic, [en línea], InterSol. 1996-2004, [última consulta el: 19 del 08del 2004. Disponible en: 
no se han obtenido datos estadísticos para todos los indicadores a la hora de recoger información.

La mayoría de los estudios presentan información muy general de los países. En general, la literatura encontrada contiene información desactualizada y se refiere básicamente al funcionamiento de Internet en los distintos países; no habla acerca de los problemas de acceso, ni tampoco ofrece datos estadísticos. La mayoría de las páginas web que se han encontrado ofrecen la posibilidad de leer en dos o tres lenguas: inglés, francés y árabe, aunque predomina la francesa por sobre la inglesa. En el caso de Libia se encuentran algunos datos en italiano, pues en su día fue colonia Italiana. Se han encontrado también algunas páginas web en las que se necesita un password para entrar en ellas, ya que o bien son universidades con campus virtuales, o bien son sitios de pago, y por eso no las hemos podido consultar.

También se han localizado diferentes estadísticas de las que se han podido extraer algunos datos, aunque no de manera homogénea para todos los países, pues o bien el país no había sido analizado o carecía en algún caso del propio dato. Por otra parte cuando se han obtenido datos diferentes, no se han eliminado por entender que los parámetros para su obtención han podido ser distintos.

La mayor fuente de información de datos estadísticos, en nuestro caso, ha sido proporcionada por la Unión Internacional de Telecomunicaciones (ITU), a la que se le pidió la posibilidad de obtener aquellos datos que ellos no tenían actualizados en la web, y la cual también nos informó cómo habían calculado algunos de ellos. Otra fuente de información consultada fue la CIA $F_{a c t b o o k}{ }^{7}$ que nos proporcionó datos sobre cada uno de estos países. No hay información sobre todos los años, y éstos no son siempre los mismos y la actualización de ellos es muy variada. También se solicitó información acerca de cómo habían sido efectuados los cálculos y la posibilidad de obtener algunos de ellos más actualizados, pero no obtuvimos respuesta.

\section{Resultados}

\subsection{Los Indicadores Socioeconómicos}

A continuación se analizan los factores socioeconómicos mencionados con anterioridad.

7 CIA - The World Factbook, [en línea], Central Intelligence Agency, 2004, [última consulta el: 21 del 11 del 2004]. Disponible en:

<http://www.cia.gov/cia/publications/factbook/index.html>. 


\subsubsection{Población}

La distribución de población es muy variada en estos países. En la Figura 4 se aprecia el crecimiento de los últimos años en términos absolutos.

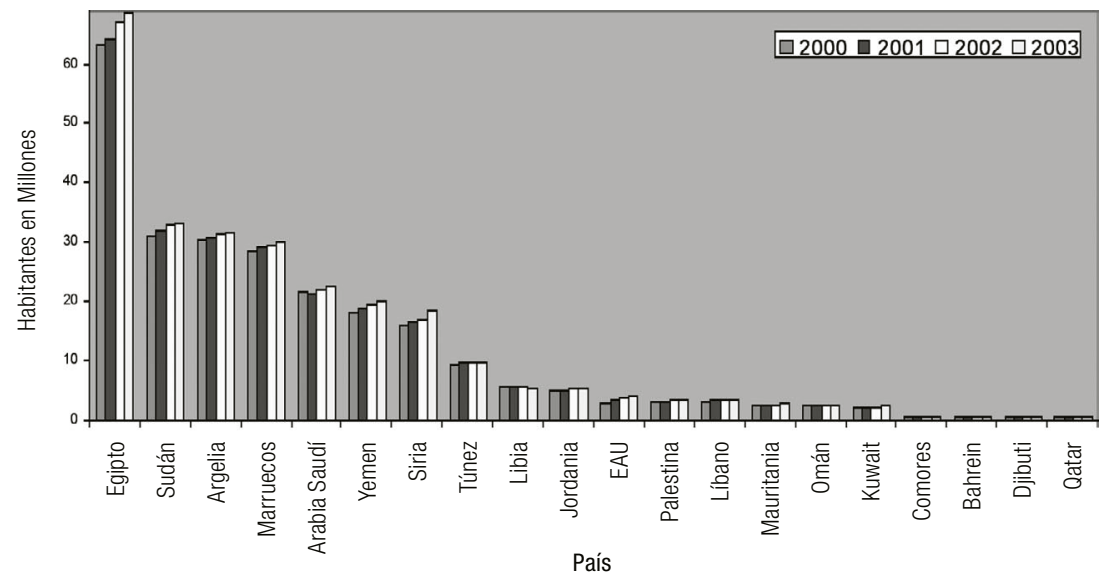

Fig. 4. Población en los últimos años.

Fuente: Elaboración propia a partir de los datos de ITU.

\subsubsection{Renta Per Cápita}

El Producto Interno Bruto (PIB) de los países de la región en la Figura 5 demuestra que Arabia Saudí es el país con mayor PIB, sostenido exclusivamente por la exportación petrolífera. Con mucha diferencia lo sigue Egipto, que es el centro geográfico de los países de la región, y cuya mayor fuente de ingresos es el turismo. En el polo contrario, tenemos que los países menos ricos son, por un lado, Dijbuti, que sobrevive gracias a las ayudas recibidas, y por el otro Comores, que como ya se ha dicho es un archipiélago pequeño cuya importancia económica es muy pequeña. 


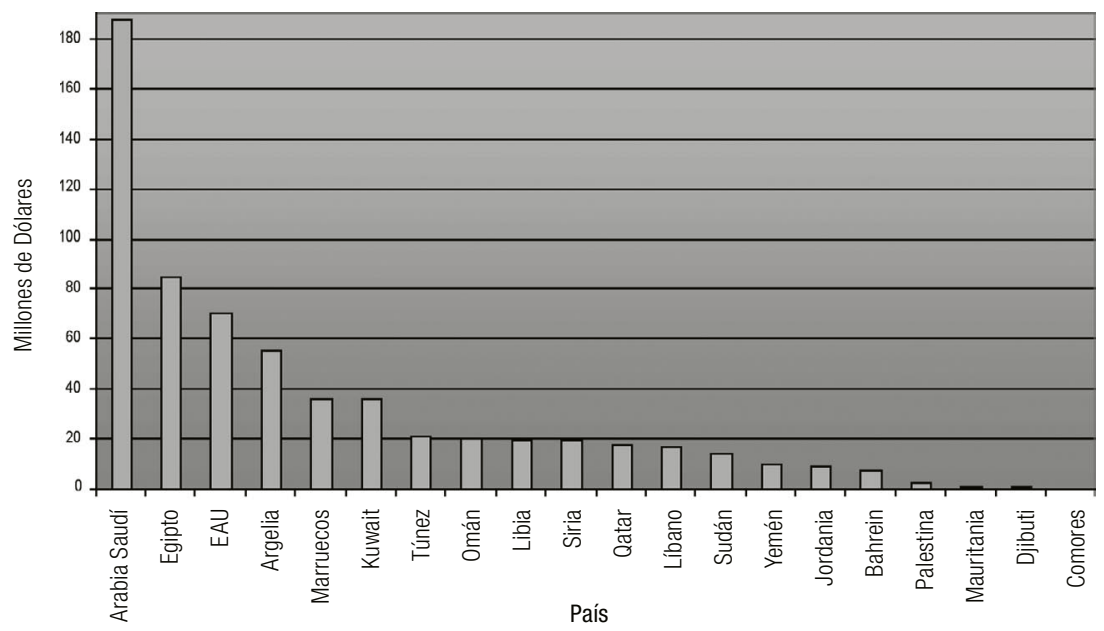

Fig. 5. Riqueza de los países en año 2002. Fuente: Elaboración propia a partir de los datos de ITU.

Podemos agrupar los países árabes en tres niveles (A, B y C), según su riqueza. En primer lugar tenemos los países del grupo A, que coinciden con los países del Golfo: Qatar, EAU, Kuwait, Bahrein, Arabia Saudí, y Omán, y que disfrutan de una renta per cápita muy elevada; entre ellos destaca, de manera negativa, Omán, ya que se puede encontrar con respecto a otros parámetros dentro del nivel medio, pues ha sido uno de los últimos países de la zona del Golfo que introdujo las tecnologías de la información. En segundo lugar están los países de renta media, grupo B, de origen bastante diverso: Líbano, Libia, Túnez, Argelia, Jordania, Egipto, Marruecos y Siria; en este grupo encontramos a países con diferentes grados de modernización y desarrollo de las TIC en función del potencial económico de cada uno de ellos.

Finalmente están los países de renta más baja, grupo C: Djibuti, Palestina, Yemen, Sudán, Mauritania y Comores. Es importante también señalar que la mayoría de estos países son países subdesarrollados, a excepción de Palestina e Iraq. Además ha de tenerse en cuenta el caso particular de Palestina, que aun estando dentro de este nivel bajo de poder adquisitivo, en muchos otros factores se comporta como un país del grupo B. 


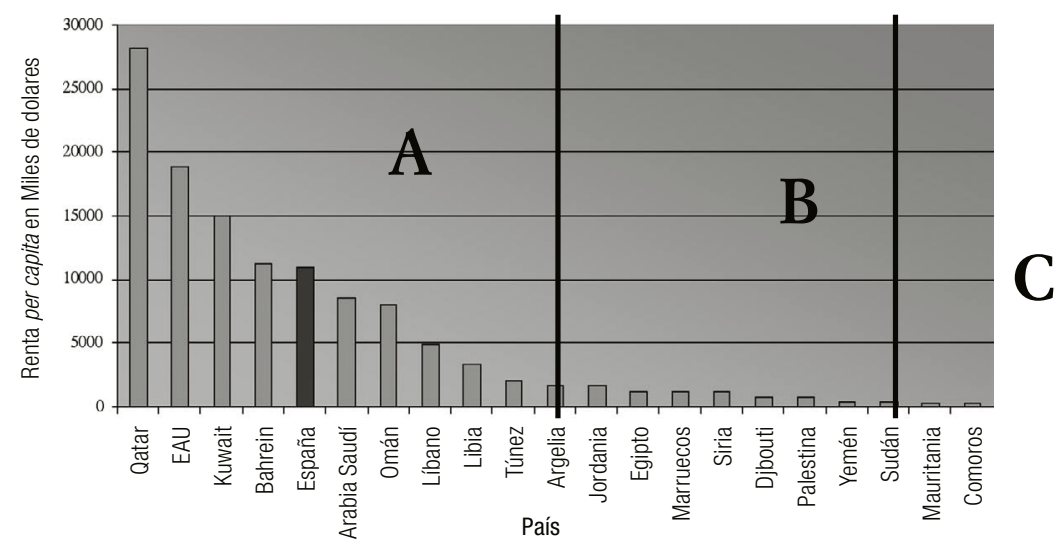

Fig. 6. Renta per cápita de los Países de la Región MENA y España en el 2002. Fuente: Elaboración propia a partir de los datos de ITU.

Como puede apreciarse en la Figura 6, en la que se compara la renta per cápita de España, como ejemplo de país europeo en contraposición a los Países Árabes, los países que superan la renta española son precisamente aquellos que se encuentran en el grupo A.

\subsubsection{Alfabetización}

Otro factor determinante del que en gran medida depende la evolución de las TIC, y más concretamente el uso de Internet, es el nivel de alfabetización del país. A este respecto puede apreciarse en la Figura 7 (página derecha) que en casi todos los países, más de la mitad de la población tiene estudios básicos, con excepción de tres países: Iraq, Somalia y Mauritania, que por los motivos expuestos anteriormente, carecen de ella.

La Figura 7, corresponde al índice de alfabetización entre los adultos mayores de 15 años. Se aprecia un gran nivel educativo en Jordania, debido al interés que el gobierno ha mostrado hacia la cultura como bien generador de economía, aun tratándose de un país con renta per cápita media baja. También ocurre lo mismo con el Líbano. Sin embargo, esto no explica que quien tenga el nivel de renta per cápita alto deba tener un nivel cultural elevado. Esto puede apreciarse en el caso de los países del grupo A, en particular Arabia Saudí, los EAU y Omán, que presentan índices de alfabetización relativamente modestos. Esto es resultado de una inversión insuficiente en educación a pesar de la riqueza generada por el petróleo. No obstante, tanto en Qatar como en EAU tienen mayor formación las mujeres que los hombres, ya que a éstas les resultas más fácil encontrar trabajo si están preparadas intelectualmente. 


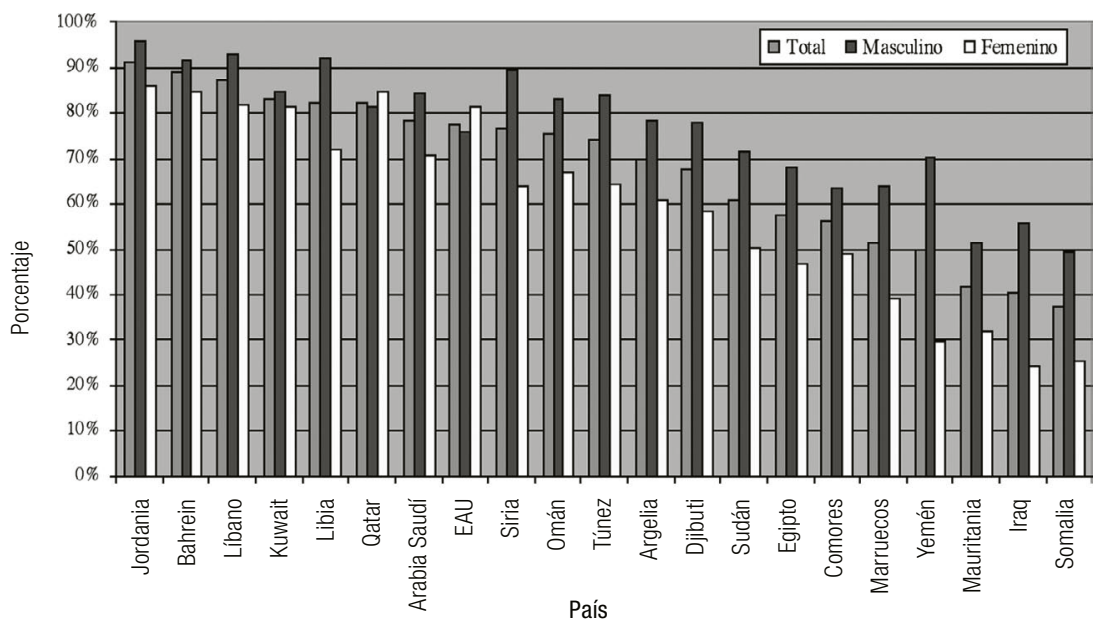

Fig. 7. Porcentaje de población alfabetizada en el 2003.

Fuente: Elaboración propia a partir de los datos de la CIA.

Los países más pobres, como el caso de Yemen o Mauritania, presentan tasas muy bajas de alfabetización, y en Iraq la situación se ha deteriorado de forma dramática a causa del embargo de los años 90, y la posterior guerra de principios de esta década. Entre los países menos alfabetizados cabe destacar la diferencia entre los índices de alfabetización de los hombres (relativamente comparable a la de los demás países) y las mujeres (muy baja en países como Somalia, Marruecos o el propio Iraq).

\subsection{Los Indicadores Tecnológicos}

Los indicadores tecnológicos reflejan la evolución de cada país con respecto a la tecnología teniendo presente que no todos los habitantes tienen acceso a los bienes de consumo que ésta proporciona. Algunos indicadores se han calculado con base en la renta per cápita de cada uno de los países, mientras que otros se han calculado con base al número de habitantes.

\subsubsection{Lineas Telefónicas}

El primer factor a analizar son las líneas telefónicas existentes en cada país. Como puede observarse en La Figura 8 (pagina siguiente), casi todos los países del Golfo cuentan con un mayor número de líneas telefónicas por ciudadano. A la cabeza se encuentra EAU, aunque como se ve tuvo un descenso considerable; EAU, junto con Marruecos, Bahrein, Kuwait y, en el último año, Jordania, son los únicos en los que descendió el número de teléfonos fijos y 
adquirió mayor importancia la telefonía móvil, que ofrece mejores prestaciones de servicios que la telefonía fija e incluso ha reducido el precio de la telefonía móvil.

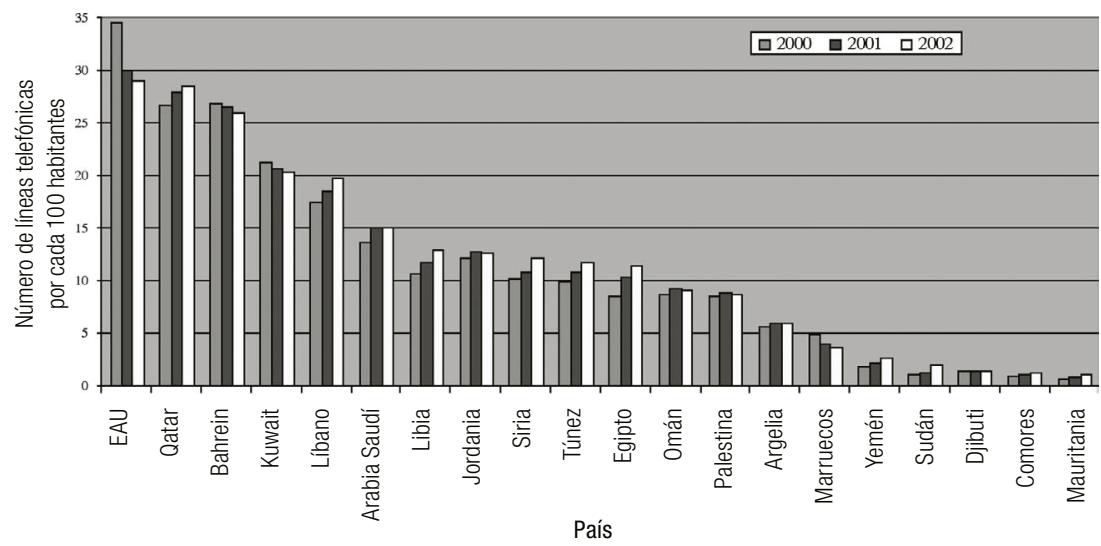

Fig. 8. Aumento del número de líneas telefónicas por cada 100 habitantes durante los años 2000 al 2002. Fuente: Elaboración propia a partir de los datos de ITU.

En Líbano puede apreciarse también un aumento considerable de teléfonos, debido a la liberación del sector de las telecomunicaciones. Como era de esperar, todos los países con nivel económico más bajo se encuentran en la parte final de la gráfica: Yemén, Sudán, Djibuti, Comores y Mauritania.

\subsubsection{Ordenadores}

Otro parámetro que se ha estudiado ha sido el número de ordenadores personales, ya que sin ellos no es posible la conexión a Internet. La Figura 9 (pagina derecha) muestra que los países con renta más elevada son también aquellos que tienen más ordenadores por habitante, con Qatar a la cabeza.

Destaca el espectacular aumento del número de ordenadores en Arabia Saudí, a causa de la reducción de precios en el 2002. Una vez más, Omán se encuentra al final de los países ricos, siendo incluso superado por Líbano. Los países de renta media y baja tienen un índice muy bajo de ordenador por habitante, como es el caso de Egipto, Argelia y Yemén, con menos de dos ordenadores por cada cien habitantes. 


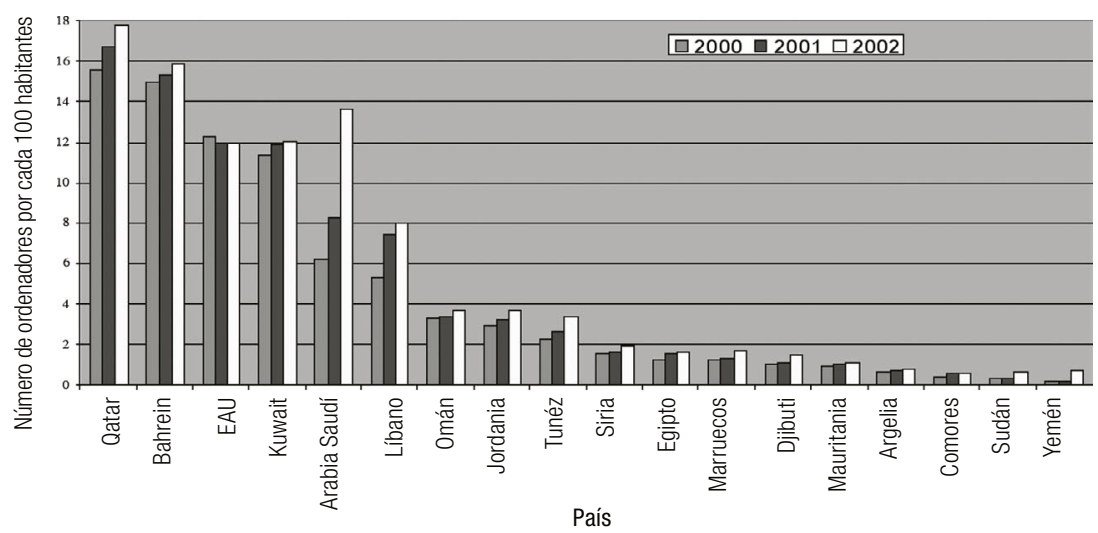

Fig. 9. Crecimiento de ordenadores por cada 100 habitantes desde el 2000 al 2002.

Fuente: Elaboración propia a partir de los datos de ITU.

\subsubsection{Proveedores}

Aunque se han identificado una serie de proveedores en los diferentes países de la región, esto no indica que sean los únicos existentes, ya que en la mayoría de los países en los que solamente existe un proveedor, éste suele ser un proveedor estatal que le da suministro a otros.

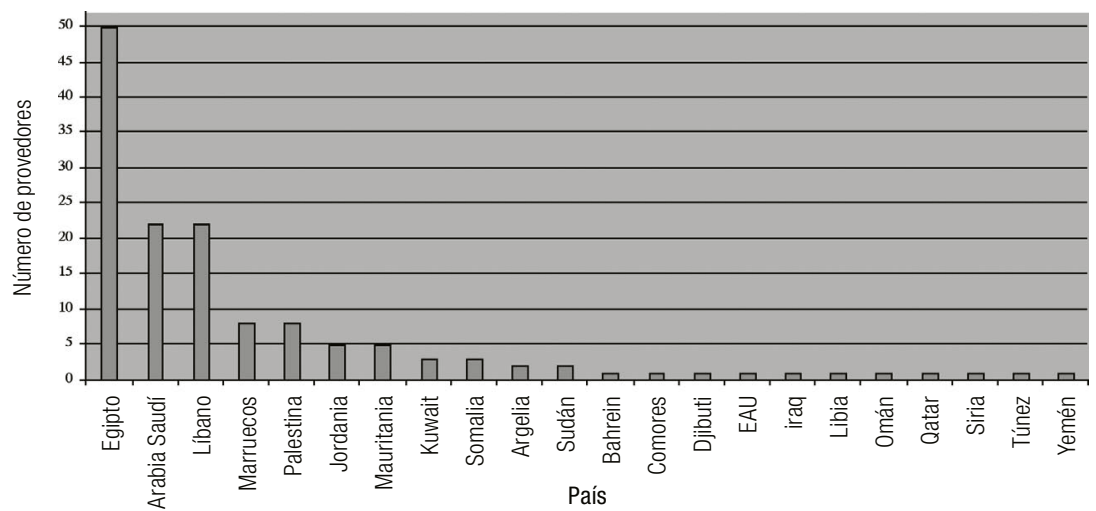

Fig. 10. Número de proveedores en cada uno de los países.

Fuente: Elaboración propia a partir de los datos de MidEastWeb ${ }^{8}$

La Figura 10 refleja el número de proveedores en los países estudiados. No es sorprendente encontrar a Egipto en el primer lugar, puesto que se trata

8 Mideast At A Glance: Countries. [en línea], Middle East Countries at a Glance. 1999, [última consulta el: 19 del 07 del 2004]. Disponible en:

<http://www.mideastweb.org/countries.htm>. 
del país más poblado de la región. Destaca el número de países en los que el sector de telecomunicaciones se encuentra en situación de monopolio, la cual incluye tanto a países ricos (Qatar, por ejemplo) como pobres (Yemén, Iraq, etcétera).

\subsubsection{Servidores}

En cuanto a los servidores presentes en cada uno de los países sobre los que se han podido obtener datos, pueden verse las cifras obtenidas en el gráfico siguiente (Figura 11).

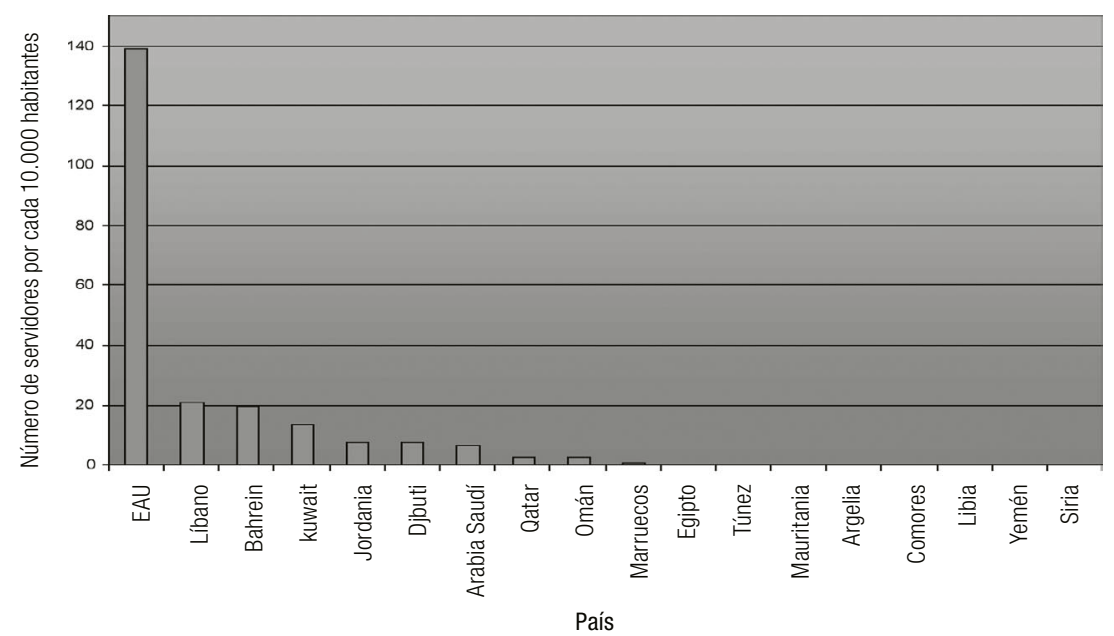

Fig. 11. Número de servidores por cada10.000 habitantes en el año 2003. Fuente: Elaboración propia a partir de los datos de ITU.

En cuanto a servidores, destaca la excelente posición de los EAU, los cuales ofrecen un excelente servicio a los clientes debido a que el mercado está relativamente liberalizado. En comparación, los demás países de este estudio cuentan con un número reducido de servidores, sobre todo Túnez (el primer país de la región donde apareció Internet), o Siria (que ha sido uno de los últimos países de la región en introducir Internet).

Una vez más, los medios económicos no le garantizan un mejor servicio al usuario, puesto que Omán y Qatar se encuentran entre los países que tienen un menor número de servidores de Internet (aunque en el caso de este último debe tenerse en cuenta que se trata de un país pequeño, y que tiene pocos habitantes). Es llamativo que Dijbuti sea uno de los países con mayor número de servidores por habitante a pesar de no ser uno de los más ricos, lo 
que quizá se deba a haber modernizado y actualizado sus servicios de telecomunicaciones.

\subsubsection{Usuarios}

Los usuarios de Internet por cada 10.000 habitantes se reflejan en la Figura 12. El número de usuarios de Internet se considera uno de los indicadores tecnológicos más importantes. El gran número de usuarios que se aprecia en EAU se debe fundamentalmente al gran número de servidores existentes en este país. También destaca el espectacular crecimiento que ha experimentado Bahrein, que pasó de 630,3 a 2.034 usuarios por cada 10.000 habitantes. Un crecimiento superior al triple del año anterior. En este caso, sin embargo el crecimiento no se debió ni a los servidores ni a los proveedores, sino más bien a la unión de cuatro factores ya analizados: densidad de población, alfabetización, renta per cápita y al número elevado de ordenadores por habitante.

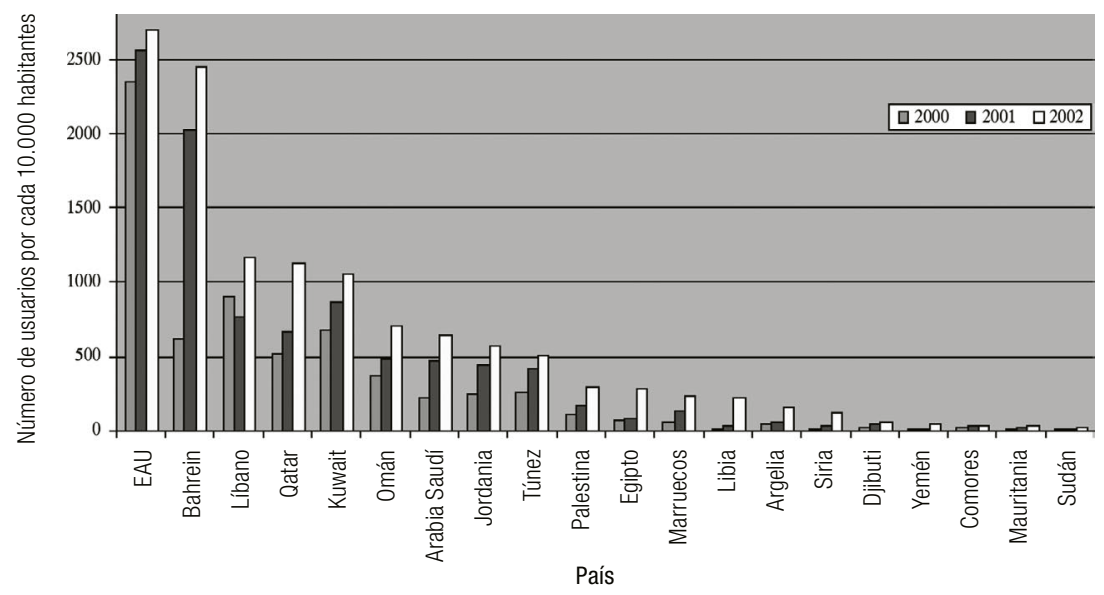

Fig. 12. Número de usuarios de Internet por cada 10.000 habitantes en los años 2000-2002. Fuente: Elaboración propia a partir de los datos de ITU.

Por otra parte Líbano tiene más usuarios que otros países con mayor renta per cápita debido a la liberalización del sector de las tecnologías, así como a la mejora de las infraestructuras. Como viene siendo habitual, se aprecia en el gráfico que los países con menor número de usuarios de Internet son los más pobres económicamente. Asimismo, Kuwait y Qatar han sufrido un alto crecimiento en el último año, y por su parte EAU ha sido el país que menos ha crecido. Sin embargo, fue éste el país con un mayor número de usuarios iniciales, debido a la gran difusión de los cibercafés y a la conexión a Internet 
de muchas escuelas y de todas las universidades, así como a la baja de precios de la telefonía fija en un 34\% en el 2001.

\subsection{Análisis detallado de los datos en función de algunos parámetros tecnológicos}

Para una mayor facilidad a la hora de identificar el país con su código de dominio se han usado los códigos de los países según la norma ISO 3166, siendo ésta la relación alfabética:

Tabla II: Relación de los países con sus códigos de dominio.

\begin{tabular}{|l|c|}
\hline Arabia Saudí & sa \\
\hline Argelia & $\mathrm{dz}$ \\
\hline Bahrein & $\mathrm{bh}$ \\
\hline Comores & $\mathrm{km}$ \\
\hline Dijbuti & $\mathrm{dj}$ \\
\hline Egipto & $\mathrm{eg}$ \\
\hline Emiratos Árabes Unidos (EAU) & $\mathrm{ae}$ \\
\hline Iraq & iq \\
\hline Jordania & jo \\
\hline Kuwait & $\mathrm{kw}$ \\
\hline Líbano & $\mathrm{lb}$ \\
\hline Libia & $\mathrm{ly}$ \\
\hline Marruecos & $\mathrm{ma}$ \\
\hline Mauritania & $\mathrm{mr}$ \\
\hline Omán & om \\
\hline Palestina & $\mathrm{ps}$ \\
\hline Qatar & qa \\
\hline Siria & sy \\
\hline Somalia & so \\
\hline Sudan & sd \\
\hline Túnez & ye \\
\hline Yemen & \\
\hline
\end{tabular}

Fuente: ISO 3166

\subsubsection{Población total en millones de habitantes por miles de} lineas telefónicas en el año 2002

En la Figura 13, se representa la cantidad de líneas telefónicas en relación con la población. Aquí destaca de manera sobresaliente Egipto (EG), Arabia Saudí (SA) y Siria (SY), países que tienen más líneas telefónicas que la media de aquellos que tienen mayor población. En este mismo grupo de países con mayor población, Argelia (DZ), Marruecos (MA), Sudán (SD) y Yemén son los 
que disponen de un número más bien escaso de líneas telefónicas, en especial en Sudán (SD).

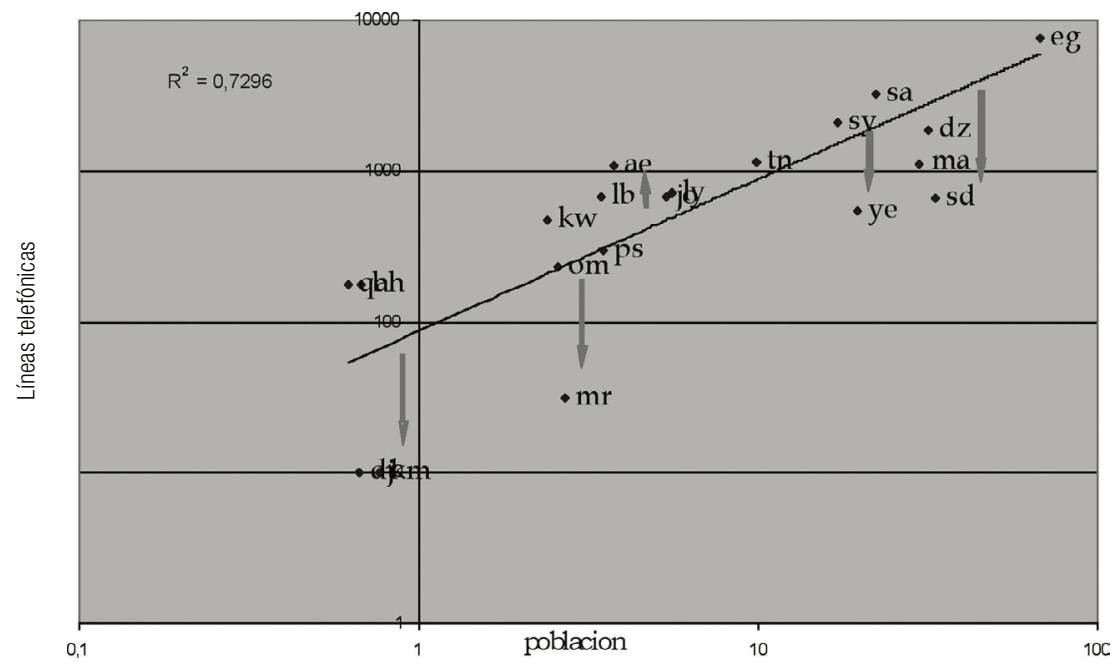

Fig. 13. Líneas telefónicas en función de la población total en el año 2002.

Fuente: Elaboración propia a partir de los datos de ITU.

Se aprecia que los países más próximos a la media son Omán (OM) y Palestina (PS), y destacan en este grupo Kuwait (KW), Líbano (LB) y, de manera sobresaliente EAU (AE). Finalmente se observa un tercer grupo de países cuya población es más baja y, que sin embargo tienen gran número de líneas telefónicas, como es el caso de Qatar (QA) y Bahrein (BH); en el lado opuesto se encuentran Dijbuti (DJ) y Comores (KM). También es llamativo el caso de Mauritania (MR), cuya población se encuentra en el segundo grupo, pero que por el número de líneas telefónicas se encuentra más próximo a este tercer grupo donde escasean las líneas telefónicas.

\subsubsection{Lineas Telefónicas por cada 100 habitantes en función de la renta per cápita en el año 2002}

En la Figura 14, donde se representan las líneas telefónicas en función de la renta per cápita, pueden apreciarse tres grandes bloques de países. El primero lo constituyen aquellos cuyas rentas per cápita son menores y que se corresponden con el grupo C mencionado anteriormente. Éste se encuentra por debajo de la línea de tendencia existente, y tiene menor número de líneas telefónicas que el resto de los países. Aquí destaca el caso de Argelia (DZ), 
que es el país más próximo a la línea de tendencia; pero encontramos también a Marruecos (MA), ambos pertenecen a los países de economía media pero, sin embargo, se encuentra muy por debajo de la tendencia.

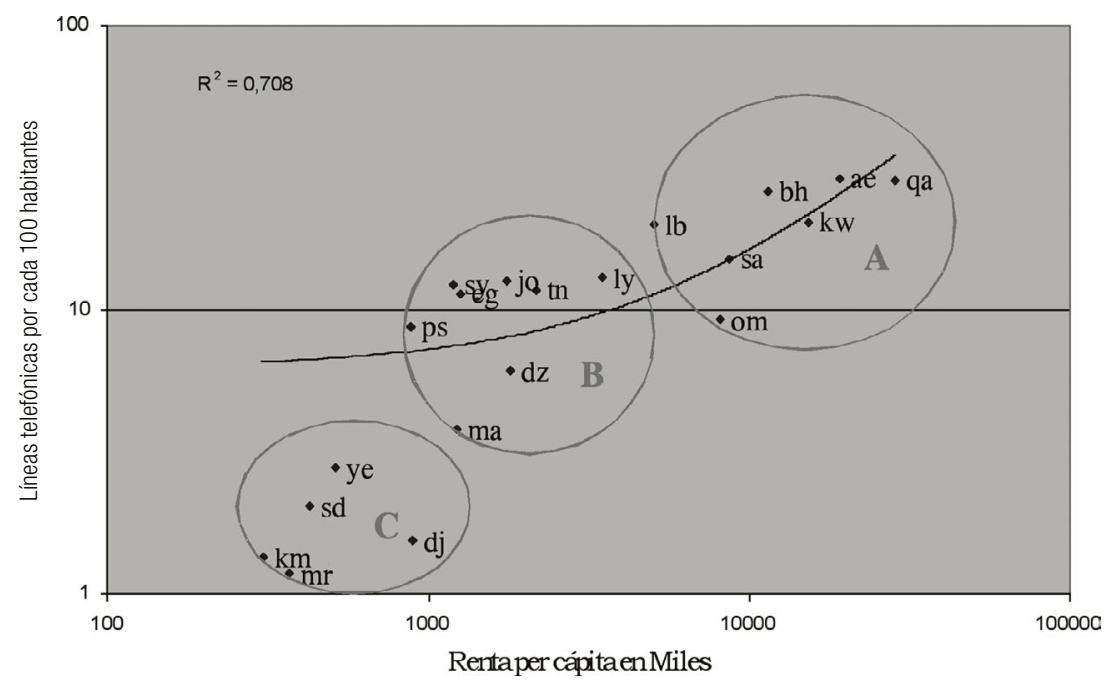

Fig. 14. Líneas telefónicas en función de la renta per capita en el año 2002.

Fuente: Elaboración propia a partir de los datos de ITU.

En la parte intermedia se encuentra el grupo B, países que se sitúan por encima de la línea de tendencia, entre los cuales destaca sobre todo Líbano (LB), pues tiene un mayor número de líneas telefónicas que la media de los países por haber liberalizado el sector y, como se apuntaba anteriormente, podría, en este sentido ser considerado como parte de los países del grupo A. En este grupo se encuentra también Palestina (PS), la que en función de su renta per cápita debiera estar en el grupo C, al superar la media de los países de su entorno debido a las ayudas recibidas no sólo por parte de los países árabes sino también de occidente, en especial de las Naciones Unidas. Finalmente, el grupo A, formado por quienes se encuentran más próximos a la media, con excepción de Omán (OM) que, como ya mencionamos, fue uno de los últimos países en introducir las TIC en su territorio.

\subsubsection{Renta per cápita en miles de dólares por número de ordenadores por cada 100 habitantes en el año 2002}

En este caso se aprecia (en la Figura 15) nuevamente la separación de los países en función de su renta per cápita. Entre los países del grupo A, se encuentra 
nuevamente Líbano (LB), porque tiene además un gran número de ordenadores y que supera la media de los países no sólo de su grupo. Se aprecia también que Omán (OM) se encuentra muy por debajo de la media en cuanto al número de ordenadores.

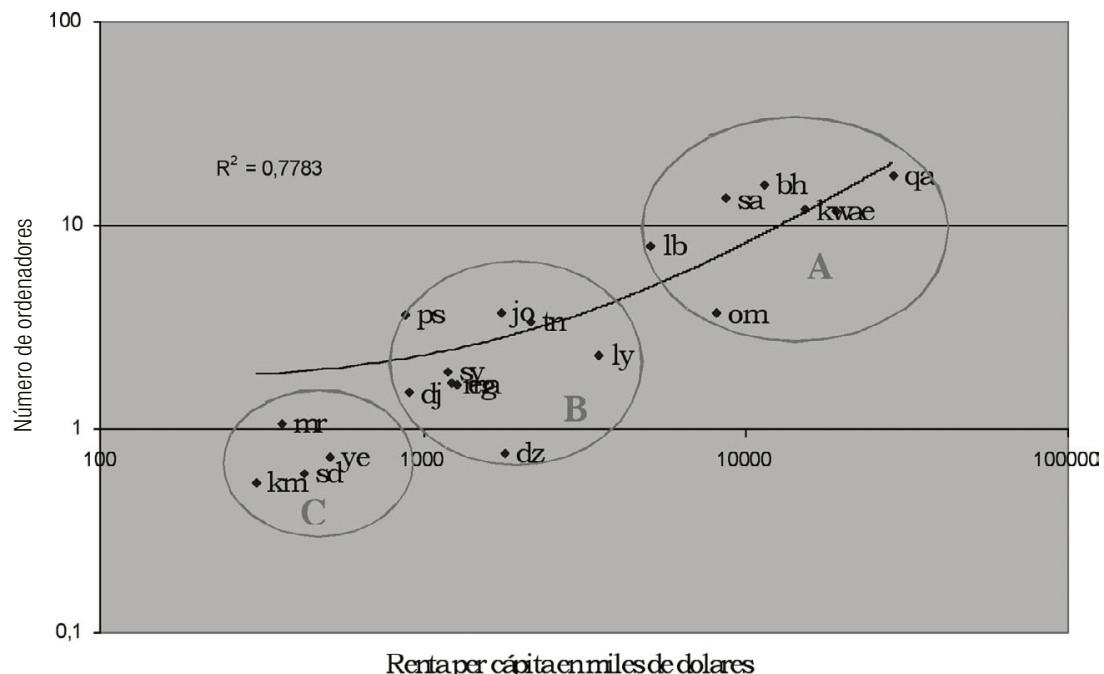

Fig. 15. Número de ordenadores en función de la renta per cápita en el año 2002. Fuente: Elaboración propia a partir de los datos de ITU.

En el caso de los países del grupo B, sobresale Palestina, que en principio pertenecería al grupo C. En este caso, como ocurre con Líbano (LB), también tiene un nivel muy superior a la media. Argelia (DZ) destaca negativamente respecto al número de ordenadores en este grupo B. Por último, se ha encontrado que en aquellos países del grupo $\mathrm{C}$, el número de ordenadores también es el menor. A excepción de Djibuti (DJ) que experimentó un salto cuantitativo en el número de ordenadores por cada 100 habitantes así como en el número de usuarios de Internet; ha de tenerse en cuenta que, adicionalmente, que es el primer país del grupo C.

\subsubsection{Renta per cápita en miles de dólares por usuarios por cada 10.000 habitantes en el año 2002}

En la Figura 16 se observan nuevamente tres grandes grupos: en el grupo A se encuentra Bahrein (BH), país que tiene un número de usuarios mayor a la renta per cápita. En este caso Líbano (LB) se encuentra en este grupo A, como ya se ha mencionado con anterioridad, al tener un nivel de usuarios mayor a 
la media. Dentro de este grupo destaca negativamente Qatar (QA), pues se encuentra muy por debajo de la media de estos países.

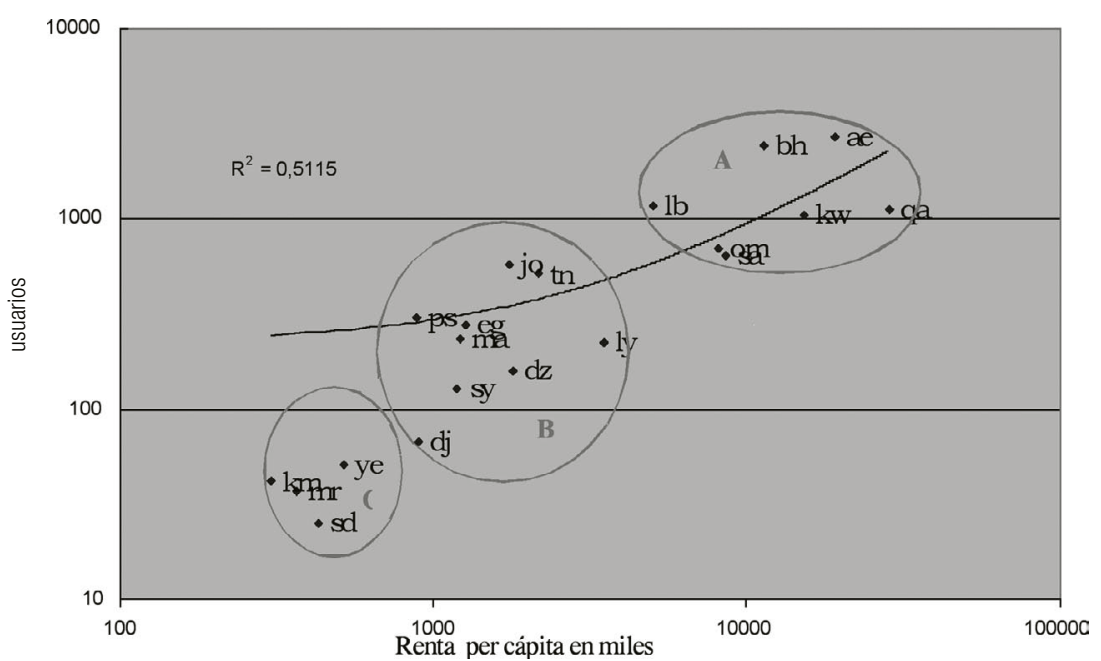

Fig. 16. Número de Usuarios en función de la renta per cápita en el año 2002.

Fuente: Elaboración propia a partir de los datos de ITU.

Con relación a los países del grupo B, la mayoría de éstos se encuentran por debajo de la línea de tendencia, en especial Siria (SY), Libia (LY) y Argelia (DZ). Llama la atención en este grupo la presencia de Palestina, ya que aun perteneciendo al grupo $\mathrm{C}$, se encuentra un poquito por encima de la línea de tendencia. Finalmente, debe destacarse en el grupo $\mathrm{C}$ que los usuarios de Internet son muy escasos, sobre todo en Sudán (SD).

\section{Conclusiones}

Cómo conclusiones finales del trabajo, podemos afirmar que:

1. Gran parte de los países de la región tienen un sistema económico centralizado, especialmente en las áreas de infraestructura y servicios. Esto produce como consecuencia la eliminación de la competencia y, por tanto, del desarrollo en términos generales.

2. Muchos de estos países han padecido una falta de seguridad, por circunstancias tanto internas como externas. A consecuencia de esto, suelen utilizar los recursos originalmente destinados a su infraestructura pública, hacia fines estratégicos y militares. 
3. Un caso excepcional serían los Emiratos Árabes Unidos (EUA), los cuales han obtenido niveles elevados en el mundo de las TIC, con la creación de un mercado de materiales electrónicos y de diversos proyectos en el campo de ciencias de la información automatizadas. Otros países que han llevado a cabo un plan similar, aunque con resultados menos satisfactorios, serían Qatar y Egipto.

4. Sigue siendo el caso de los EUA, junto con Bahrein, un caso donde se da el monopolio de tecnologías de información, al haber eliminado la competencia, por un lado, y al haber logrado un desarrollo notable en el sector de comunicaciones, por el otro. Esto se explica por la singularidad de los casos, debido al buen nivel económico de los EUA, más la reducida extensión de los países que la constituyen y su baja densidad de población.

5. El desarrollo de Internet como fuente de información afronta otros problemas, tales como el inconveniente del propio idioma dentro de la red (no hay suficientes recursos en árabe), junto con la gran subida de precios generalizada de los ordenadores y la dificultad de su uso que existe por parte de los usuarios.

6. El nivel de desarrollo de las TIC depende en gran medida de la renta per cápita.

7. Hay una relación directa entre la difusión del servicio telefónico y el nivel de la renta por habitante.

8. En el caso especial del Líbano apreciamos que casi siempre aparece asociado al grupo A, donde el sector de las TIC esta más avanzado y liberalizado comparado con la renta per cápita del propio país, lo que implica que tiene un nivel elevado de las TIC en comparación con su entorno.

9. Con Palestina ocurre algo similar, ya que perteneciendo al Grupo C puede verse en muchas ocasiones incluida dentro del grupo B.

10. Por el contrario tenemos el caso de Omán que perteneciendo al grupo A, en ocasiones puede verse envuelto en el grupo B, ya que fue el ultimo país de su grupo en introducir las TIC.

11. Desde mi punto de vista, la difusión de Internet en la zona no puede relacionarse sólo con el nivel de renta per cápita, y hay que relacionarlo también con la apertura social y cultural del país, y con el papel que desempeña el estado en impulsar la difusión del servicio, así como con la toma de conciencia y la creación de un ambiente favorable para el desarrollo de la Red. 


\section{BibLIOGRAFíA 9}

1. Cumbre Mundial sobre la Sociedad de la Información, en línea], Unión Internacional de Telecomunicaciones. Actualizado el 17 de diciembre del 2004. [Última consulta el 25 del 02 del 2005] Disponible en: $<$ http://www.itu.int/wsis/index-es.html>.

2. Informe sobre el Desarrollo Mundial de las Telecomunicaciones 2003: Indicadores de acceso para la sociedad de la información, en línea]. International Telecommunication Union. 2003. [Última consulta el: 26 del 09 del 2004]. Disponible en:

<http://www.itu.int/ITU-D/ict/publications/wtdr_03/material/ WTDR03Sum_s.pdf $>$.

3. Jupitermedia Corporation. NUA internet how many online, [en línea], 2004. [Última consulta el 11 del 05 del 2004]. Disponible en: $<$ http://www.nua.com/surveys/how_many_online/>.

4. The Arabic Network for Human Rights Information. The Internet In the Arab World A New Space of Repression?: Overview, [en línea], 2003-2004; [Última consulta el 16 del 11 del 2004]. Disponible en:

<http://www.hrinfo.net/en/reports/net2004/intro.shtml>

5. Intersol The Global Advisor Newsletter - Arabic, [en línea], InterSol. 1996-2004, [última consulta el: 19 del 08del 2004. Disponible en:

$<$ http://www.intersolinc.com/newsletters/newsletter_32.htm>.

6. CIA - The World Factbook, [en línea], Central Intelligence Agency, 2004, [última consulta el: 21 del 11 del 2004]. Disponible en: $<$ http://www.cia.gov/cia/publications/factbook/index.html >.

7. Mideast At A Glance: Countries. [en línea], Middle East Countries at a Glance. 1999, [última consulta el: 19 del 07 del 2004]. Disponible en:

$<$ http://www.mideastweb.org/countries.htm>.

9 Aquella referencia precedida de “樯” implica que ha sido consultada en lengua árabe, aunque en ocasiones también puede hacerse en otra lengua 